\title{
Molecular mechanisms and the vital roles of resistin, TLR 4, and NF-KB in treating type 2 diabetic complications
}

\author{
Venkataiah Gudise ${ }^{1 *}$ (D) and Bimalendu Chowdhury ${ }^{2}$
}

\begin{abstract}
Background: Type 2 diabetes in obese ( $\geq 25$ and $\geq 30 \mathrm{~kg} / \mathrm{m}^{2}$ ) patients is the foremost cause of cardiovascular complications like stroke, osteoarthritis, cancers (endometrial, breast, ovarian, liver, kidney, colon, and prostate), and vascular complications like diabetic neuropathy, diabetic and retinopathy, and diabetic nephropathy. It is recognized as a global burden disorder with high prevalence in middle-income nations which might lead to a double burden on health care professionals. Hence, this review emphasizes on understanding the complexity and vital signaling tracts involved in diabetic complications for effective treatment.

Main body: Type 2 diabetes in overweight patients induces the creation of specific ROS that further leads to changes in cellular proliferation, hypothalamus, and fringe. The resistin, TLR4, and NF-KB signalings are mainly involved in the progression of central and fringe changes such as insulin resistance and inflammation in diabetic patients. The overexpression of these signals might lead to the rapid progression of diabetic vascular complications induced by the release of proinflammatory cytokines, chemokines, interleukins, and cyclooxygenase-mediated chemicals. Until now, there has been no curative treatment for diabetes. Therefore, to effectively treat complications of type 2 diabetes, the researchers need to concentrate on the molecular mechanisms and important signaling tracts involved.
\end{abstract}

Conclusion: In this review, we suggested the molecular mechanism of STZ-HFD induced type 2 diabetes and the vital roles of resistin, TLR4, and NF-KB signalings in central, fringe changes, and development diabetic complications for its effective treatment.

Keywords: Type 2 diabetes, STZ-HFD, Resistin, TLR4, and NF-KB signaling

\section{Background}

Diabetes is a chronic lifestyle syndrome that affects millions of the global population, and it is a significant health issue and obesity plays a vital role in developing diabetes [1]. Diabetes in obese people is a significant cause of stroke, heart attacks, renal failure, impaired vision, and amputation of the lower limb, and type $2 \mathrm{DM}$ is often correlated with obesity and physical inactivity [2].

\footnotetext{
* Correspondence: venkatpharma22@gmail.com

'Department of Pharmacology, SSJ College of Pharmacy, Vattinagulapally,

Gandipet, Hyderabad, Telangana 500075, India

Full list of author information is available at the end of the article
}

About 463 million individuals globally have suffered from diabetes and type $2 \mathrm{DM}$ is significantly increasing in middle-income nations with 4.2 million deaths [3]. This is predicted to grow by 2045 to 700 million [3]. In recent years, T2DM accounted for $90-95 \%$ of diabetic cases [4].

Therefore, the treatment approaches for T2DM must be designed to reestablish or restore the $\beta$ cells or its functions, in addition to improving insulin sensitivity [5]. This review indicates the importance of understanding the mechanism (pathophysiology) of disease patterns and specific signaling mechanisms for the discovery of new therapeutic candidates. For this, several animal

\section{Springer Open}

(- The Author(s). 2020 Open Access This article is licensed under a Creative Commons Attribution 4.0 International License, which permits use, sharing, adaptation, distribution and reproduction in any medium or format, as long as you give appropriate credit to the original author(s) and the source, provide a link to the Creative Commons licence, and indicate if changes were made. The images or other third party material in this article are included in the article's Creative Commons licence, unless indicated otherwise in a credit line to the material. If material is not included in the article's Creative Commons licence and your intended use is not permitted by statutory regulation or exceeds the permitted use, you will need to obtain permission directly from the copyright holder. To view a copy of this licence, visit http://creativecommons.org/licenses/by/4.0/. 
models (spontaneous and induced) are available for the screening of various chemicals and phytopharmaceuticals. The maximum of these induced models cannot be seen as an analogy to clinical situations and the genetic models like ZDF rat and $\mathrm{db} / \mathrm{db}$ mouse spontaneously develop T2DM similar to humans, but this spontaneous induction of T2DM is not observed in humans [6]. Moreover, these animal models are highly expensive and not easily available for the screening purposes of various novel chemicals. IR and hyperglycemia are attributed to type 2 diabetes in overweight people [7].

Among several type 2 diabetic animal models, the HFD-STZ-induced model is commonly using to discover the novel medicine from various sources (for instance, phytochemicals). In this study, we described the molecular mechanism of streptozotocin and high-fat dietinduced type $2 \mathrm{DM}$ as this model develop similar characteristics to humans [8] and the roles of resistin, TLR4, and NF- $\mathrm{KB}$ signaling tracks in diabetic complications for effective treatment of diabetic vascular complications.

\section{Main text}

\subsection{Molecular mechanism of HFD-STZ-induced diabetes}

In brief, the streptozotocin (a glucose analog cytotoxic) migrates through the GLUT2 transporter and causes DNA alkylation at the 6th position of guanine moiety of the target $\beta$ cells by transferring the $\mathrm{CH}_{3}$ group to DNA leading to DNA fragmentation [9] and protein glycosylation [10]. This results in the initiation of ADP-ribose enzyme to repair the DNA and utilization of energy as ATP which further leads to depletion of cellular ATP and NAD+ stores. This depletion consequences in the establishment of dephosphorylated proteins (substrates for xanthine oxidase) and the invention of ROS (reactive oxygen species) like oxygen and hydrogen peroxides and nitro-methyl- $N$-nitrosourea side-chain proteins, which can liberate hydroxyl and nitric oxide radicals [9].

Further, the excess hydroxyl radical migrates to the cytosol and activates the aconitase, leading to mitochondrial dysfunction and further ATP depletion, which causes the rigorous formation of ROS. All these ROS damage the plasma membrane and necrosis of $\beta$ cells to induce programmed cell death and decreased insulin availability which causes hyperglycemia [11]. Earlier studies have reported that mitochondrial DNA abnormality, protein alkylation, and glycosylation are also evident for STZ-induced depletion of NAD+, which inhibits insulin biosynthesis and its secretion [12].

This hyperglycemia or diabetes by STZ is dependent on GLUT2 in $\beta$ cells. The cytotoxicity role of STZ is still not clear. However, several in vitro and in vivo studies have stated that STZ can develop hyperglycemia due to its specific $\beta$ cell necrosis and apoptosis as a result of oxidative stress and mitochondrial dysfunction [13]. It has also been reported that the cytotoxic effects of streptozotocin are dependent on time and dose to develop oxidative stress, associated alterations of GSH redox metabolism, mitochondrial respiratory, and induction of cytochrome P450 family. This result is important in understanding STZ-induced necrosis and programmed cell death (apoptosis) and the capability of pancreatic cells to breakdown other xenobiotics in stress situations [14]. The proposed molecular mechanism of STZ-HFDinduced diabetes is represented in Fig. 1.

Streptozotocin (STZ) also might cause injury to organs, particularly the kidney, liver, and those expressing GLUT2 transporter [15]. The alkylation in $\beta$ cell has been described by using ethylating chemicals that are mildly toxic at $\mathrm{O}^{6}$-ethylguanine than at $\mathrm{O}^{6}$-methylation [9] and STZ also can affect insulin and glucose homeostasis [16].

HFD-induced obesity increased the levels of TGs (triglycerides) in circulation and in fat cells (adipose) that assist in the progress of IR (insulin resistance) and dysfunction of the adipose cell and tissue [17]. This further increases the insulin level in the blood (hyperinsulinemia) where the body tissues become insensitive to insulin and inflammation [18]. The pathophysiology linking to inflammation in metabolic illness and complications has stimulated interest in targeting inflammatory pathways to prevent or manage diabetic complications [19]. The mechanism of $\beta$ cell damage via free radical generation is not only specific for STZ. This can be applicable for other diabetogenic chemicals (for instance, alloxan monohydrate) that induce the pancreatic $\beta$ cell oxidation via free radical generation in obese individuals. Among the various methods of induction, the combination of HFD and STZ will develop similar characteristics to humans [8]. Hence, we selected this model to provide the molecular mechanism type 2 diabetes pathogenesis and to identify the various signaling pathways for effective management of diabetic complications.

\subsection{Diabetic complications via NF-kB}

Diabetic complications are linked with prolonged persistence of insulin resistance and hyperglycemia [20]. The elevated glucose level in the blood (hyperglycemia) induces the creation of ROS, AGEs (advanced glycation end-products) protein kinase-C (PKC), and AT-II due to increased polyol flux, hexamine flux, PKC activation, and accumulation of AGEs [21-23]. These intermediate biomolecules are the key factors for the activation of the NF- $\mathrm{KB}$ signaling pathway $[21,23]$. The schematic representation of diabetic complications is shown in Fig. 2.

\subsection{Diabetic neuropathy}

Diabetic peripheral neuropathy (DPN) and diabetic autonomic neuropathy (DAN) are the intensive and most 


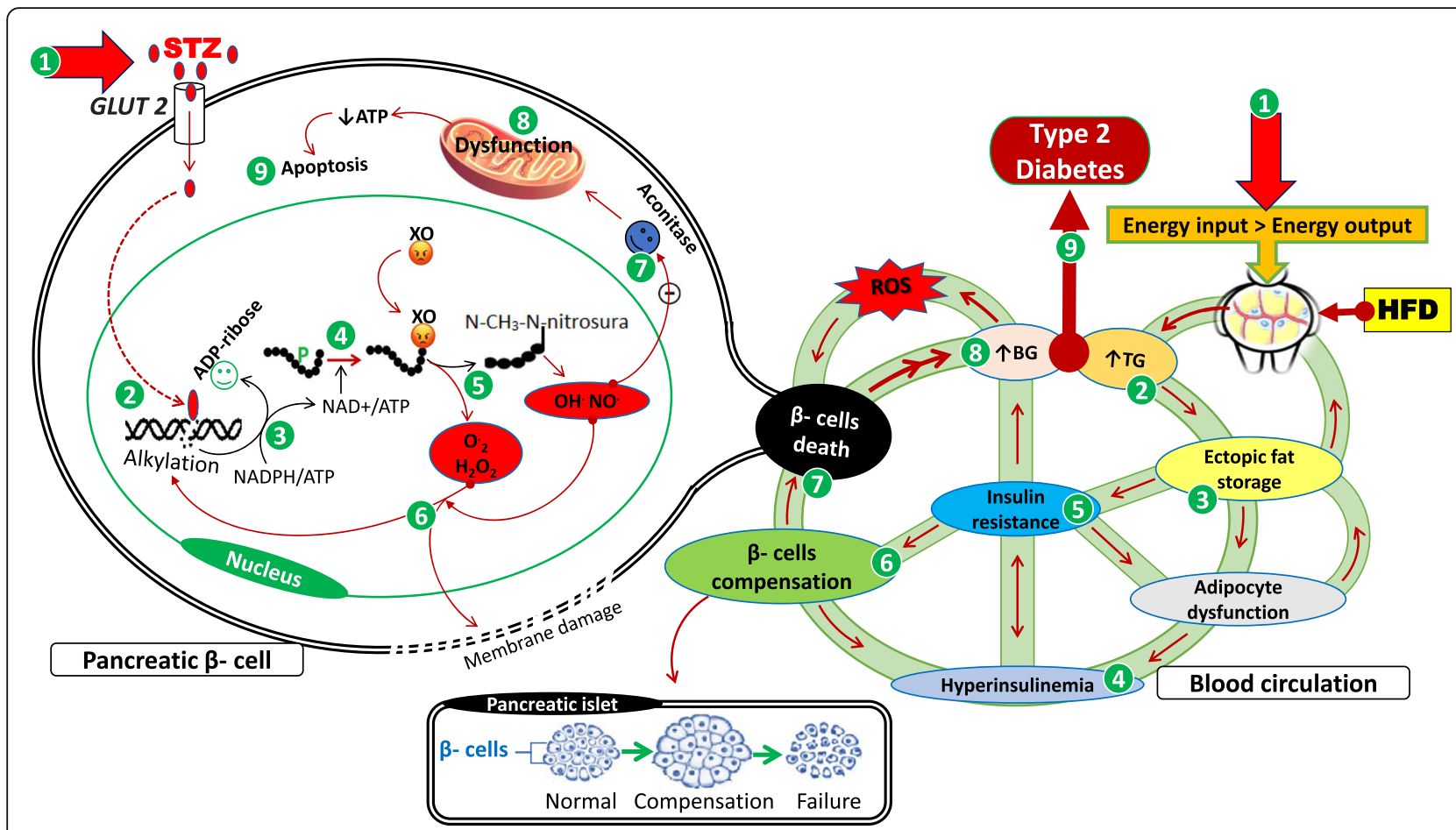

Fig. 1 Molecular mechanism of STZ-HFD induced type 2 diabetes. $\rightarrow:$ Initiation/release/damage; $\rightarrow$ : Repair/remodeling; $\oplus$ : Stimulation; $\Theta$ : Inhibition. HFD high-fat diet, TG triglycerides, BG blood glucose, ROS reactive oxygen species, GLUT2 glucose transporter 2, ADP adenosine diphosphate, NADPH nicotinamide adenine dinucleotide phosphate, APT adenosine triphosphate, NAD nicotinamide adenine dinucleotide, XO xanthine oxidase, and free radicals $\left(\mathrm{O}_{2}, \mathrm{H}_{2} \mathrm{O}_{2}, \mathrm{OH}^{*}\right.$, NO*)

severe type of vascular diabetic complication and often the principal cause of a significant rise in morbidity and mortality [24] which comprises the loss of sensation; peripheral, somatic, autonomic, and motor dysfunction; axonal thickening; loss of nerve fibers; demyelination; narrowing of the neural capillary system, and elevated MAPK that leads to nerve injury [25]; the schematic representation of pathogenesis (Fig. 2). The hyperglycemic condition triggers the AGE/RAGE formation in neurons [23], and increased HbAc1 and collagen in peripheral nerves are also an additional risk factor for diabetic nephropathy [26].

The NF- $\mathrm{kB}$ gets activated by AGE/RAGE and causes the release of TNF $\alpha[21,27]$. The augmented concentration of sorbitol can increase due to increased polyol flux by aldose reductase (AR) and the generation of ROS in neural cells [28]. The sorbitol and ROS affect nerve conduction by inhibiting sodium-potassium ATPase which delays in conduction velocity in the nerves and weakness [29]. The overstimulation of NF- $\mathrm{kB}$ also leads to the infiltration of leucocytes, reduced neural growth factors (NGFs), increased TNF $\alpha$, and IL-6 in nerve cells [30]. Simultaneously, the cyclooxygenase-2 (COX-2) is also gets activated as a result of the arachidonic acid pathway expression [31]. Elevated ICAM and NF- $\mathrm{KB}$ are noticed in sciatic-tibial nerves of diabetic rats that cause the narrowing of blood vessels and ischemia with inflammation [27]. The peroxisome proliferator-activated macromolecular receptors are declined in the nerves as a result of elevated levels of cytokines which increase neural death [32].

\subsection{Diabetic nephropathy}

Diabetic nephropathy is the principal reason for cardiovascular and chronic kidney diseases characterized by reduced GFR, microalbumin, and increased albumincreatinine ratio [33]; the schematic representation of pathogenesis (Fig. 2). Renal inflammation is a significant factor in the evolution of diabetic nephropathy. Proinflammatory cytokine molecules are accountable for the infiltration of various macrophages, monocytes, and $\mathrm{T}$ cells $[34,35]$.

Apart from this, the leucocytes can upregulate the NF$\kappa B$ in endothelial and mesangial cells $[35,36]$, thus stimulating MCP-1, leading to macrophage infiltration $[37,38]$, and increased microalbumin and renal injury $[39,40]$. The activated NF-kB also activates MAPK and enhances the response of TGF- $\beta$-activated kinase (TAK1) released from the MAPK family (MAPK3K7). The TAK1 in turn stimulates the TGF- $\beta$, which develops the fibrosis and extracellular matrix accumulation [41]. MAPK also contributes to the overexpression of various 


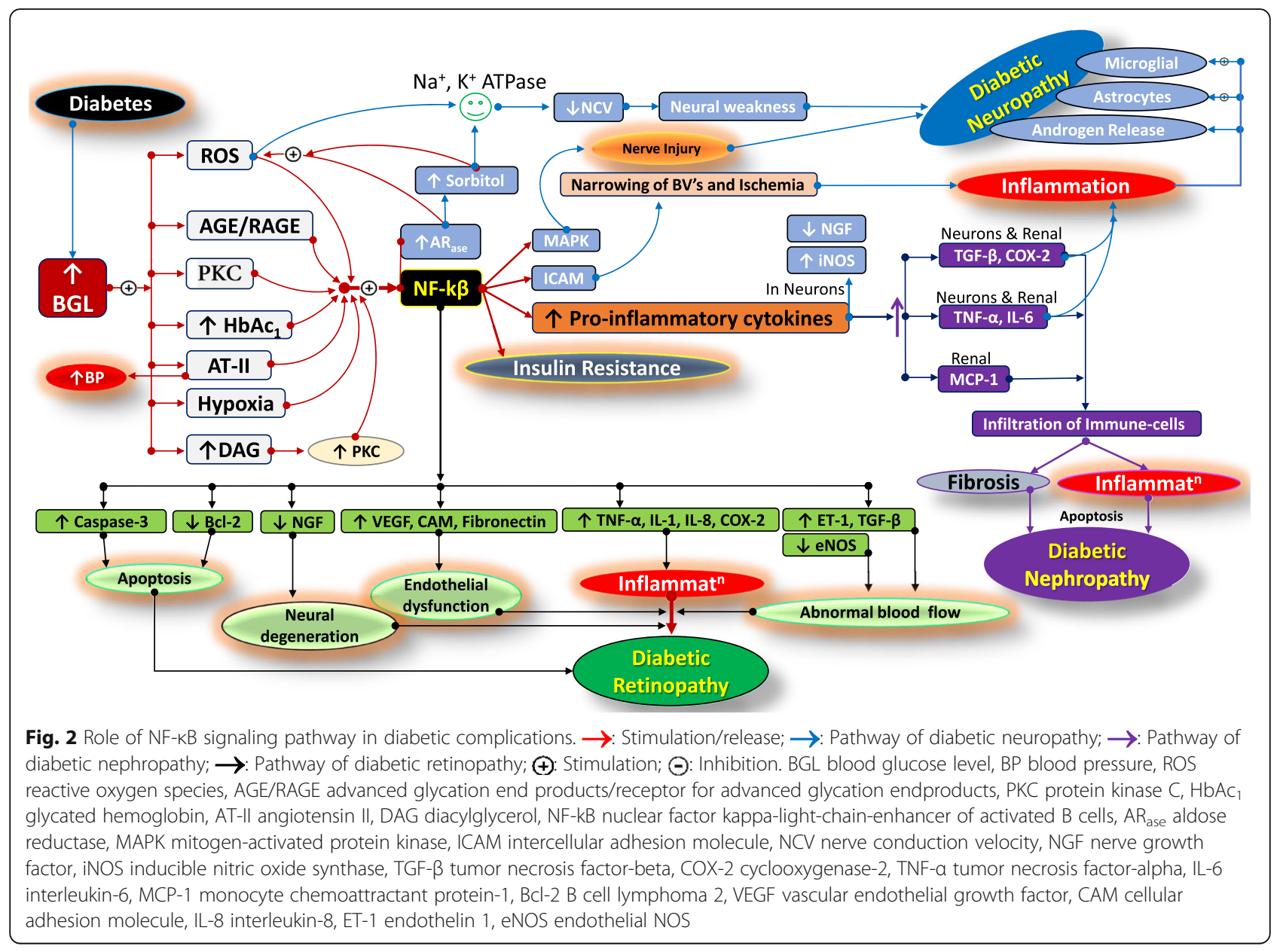

genes in releasing the cytokine molecules and intracellular adhesion molecule (ICAM), JNK, and leucocyte infiltration via NF- $\mathrm{KB}$ activation [27]. Angiotensin-II (AT-II) also upsurges as a result of AGEs and oxidized lipids in $\mathrm{DN}$ patients. This triggers the NF- $\mathrm{kB}$ via a canonical pathway, and further inflammation can develop in the kidney and cause injury [27, 42].

\subsection{Diabetic retinopathy}

Diabetic retinopathy (DR) is the greatest and specific microvascular complication and leads to blindness in diabetic patients [43]. It is indicated by pericytes loss, condensing of the capillary basement membrane, cataract, capillary cellularity, microaneurysm, and bloodretinal barrier [44]. Based on its severity, the DR is classified as proliferative-diabetic-retinopathy, nonproliferative-diabetic-retinopathy, and diabetic-macularedema [45]. As per the current information, $1 / 3$ of the estimated 285 million diabetic patients have signs of DR [46]. The development of retinopathy in the USA was estimated at $28.5 \%$ and $4.4 \%$, respectively [44]. The schematic representation of pathogenesis is shown in Fig. 2.
The mechanism of nephropathy in diabetic patients is not well understood and described, even though many proposed pathogenic have been described, viz. increased AGEs, increased ROS, PKC, aldose reductase, and cytokines $[44,47]$. The inflammation plays a major role in DR pathogenesis, and the increased levels of ROS and AGEs activate apoptotic cell death [48].

Stimulated NF-kB will increase the specific proinflammatory mediators and cytokines like tumor necrosis factors (TNFs) and IL capsase-3 that cause inflammation and cell death [49]. Further, the inflamed cells release the nitric oxide (NO) leading to the stimulation of NO synthase, which can cause the narrowing of vessels, ischemia, and flow abnormalities [50]. The release of ICAM-1, CD18, and fibronectin enhances the infiltration of leucocytes, fibrosis of retinal space, and breakdown of the retinal-blood barrier [51].

Elevated PKC and imbalance in pro-NGF and NGF further develop the neural damage or dysfunction in the retina and enhance the polyol flux and MAPK, TNF $\alpha$, and COX-2, leading to enhanced inflammation [27]. Matrix metalloproteins, especially MMP-9, also play a crucial role in angiogenesis and cell death (apoptosis) in DR. These protein levels are elevated in vitreous and 
retina in both animal models and diabetic patients [52]. Elevated ILs and MMP-9 also cause DNA alkylation and progression of DR [53].

\subsection{HFD/obesity-induced hypothalamic, fringe changes}

Obesity, a nutritional disorder, is the foremost health concern in developing countries which is the major concern in the advancement of T2D and atherosclerosis cardiovascular complications like stroke [54]. Cholesterols or triglycerides in overweight patients develop the insulin resistance and the epidemiological findings stating that insulin resistance has been related to abridged tissue sensitivity to insulin and raised pro-inflammatory mediators [55]. The schematic representation of these changes is shown in Figs. 3 and 4. These mediators are also amplified in the brain of rodents in HFD-induced metabolic inflammation, which plays a significant role in insulin resistance [56]. Primarily, this type of inflammation in the adipose tissue might lead to the release of proinflammatory biomolecules [57]. These mediators can able to alter sensitivity to insulin in the skeletal muscle, adipose tissue, and in the liver [57]. The HFD activates the local immune components in the mediobasal hypothalamus to release proinflammatory mediators which cause the neural injury [58].

The mechanism of pathogenesis of obesity-induced inflammation in the hypothalamus, and fringe insulin resistance is still not clear, but in this context, of a few potential pro-inflammatory pathways has been proposed. ROS plays a vital role in obesity and insulin resistance (IR) by inducing inflammation, oxidative stress, mitochondrial dysfunction, and ER stress [59]. JNK encourages the serine phosphorylation of IRS-1 and inhibits insulin-dependent tyrosine phosphorylation and downstream signaling $[59,60]$. The NF- $k B$ signaling activates and upregulates SOCS3, and the PTP1B proteins could be linked to inflammation and IR in the central hypothalamus [61] and the deficiency of JNK in the brain defends against HFD-induced insulin resistance [62]. The IKK- $\beta / N F-k B$ pathways also have a critical part in the

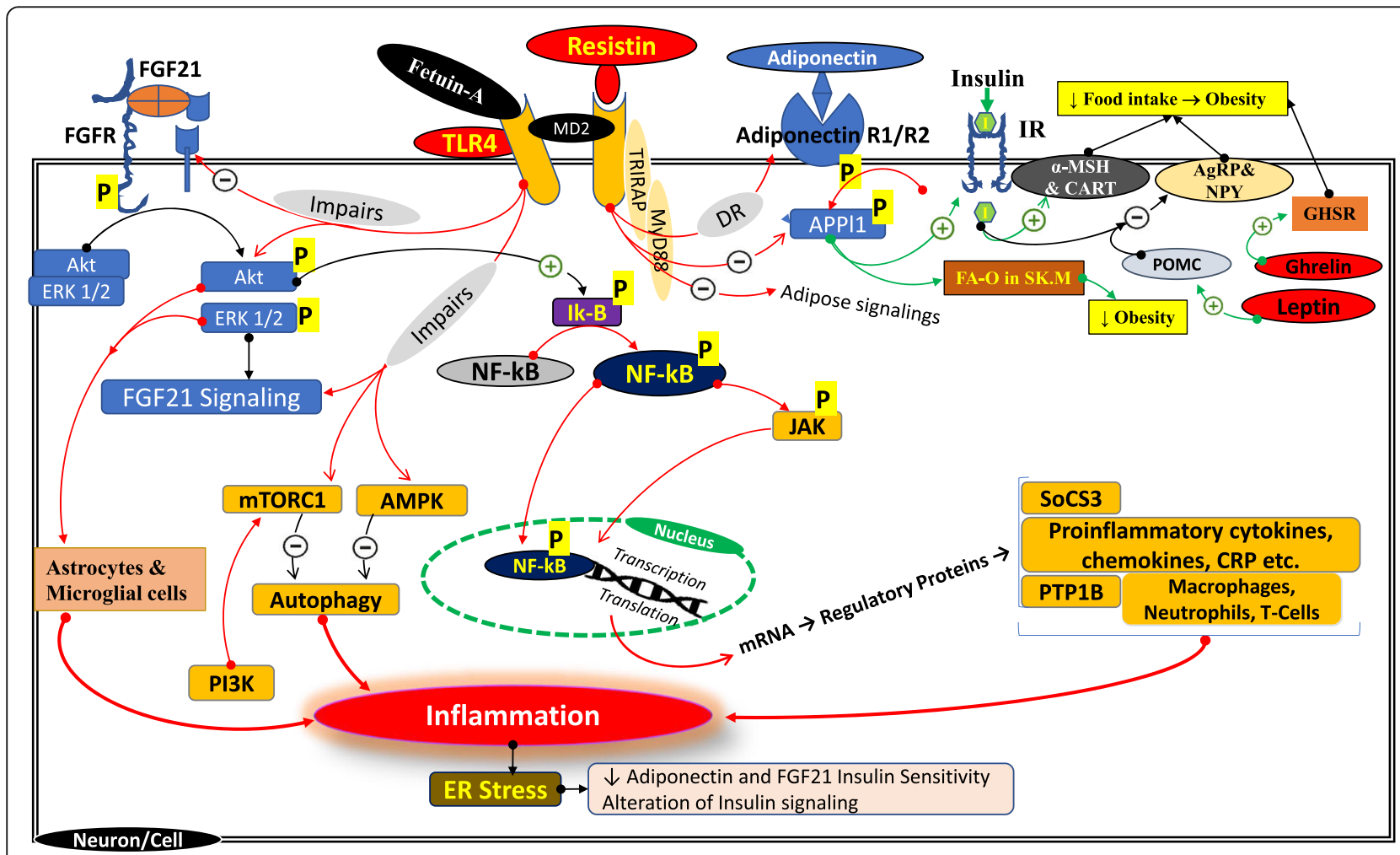

Fig. 3 Role of resistin, TLR4, and NF-KB signalings in the development of inflammation. $\rightarrow$ : Stimulation/release $\rightarrow$ : Restore/repair; $\oplus$ : Stimulation; $\Theta$ : Inhibition. FGF21 fibroblast growth factor 21, FGFR fibroblast growth factor receptor gene, Akt protein kinase B, ERK extracellular signal-regulated kinases, MD2 myeloid differentiation factor 2, TLR4 Toll-like receptor 4, TRIRAP Toll/interleukin-1 receptor domain-containing adapter protein, NF-kB nuclear factor kappa-light-chain-enhancer of activated B cells, Ik-B IkB kinase, mTORC1 Mammalian target of rapamycin complex 1, AMPK AMP-activated protein kinase, PI3K phosphatidylinositol-3-kinase, JAK c-Jun N-terminal kinases, MyD88 myeloid differentiation primary response 88 , DR diabetic retinopathy, APPI1 adaptor protein, phosphotyrosine interacting, IR insulin resistance, a-MSH \& CART amelanocyte-stimulating hormone \& cocaine and amphetamine-regulated transcript, AgRP\& NPY Agouti-related protein \& neuropeptide Y, GHSR growth hormone secretagogue receptor, POMC proopiomelanocortin, FA-O in SK.M skeletal muscle lipid oxidation, SoCS3 suppressor of cytokine signaling 3, PTP1B protein tyrosine phosphatase 1B, ER endoplasmic reticulum 


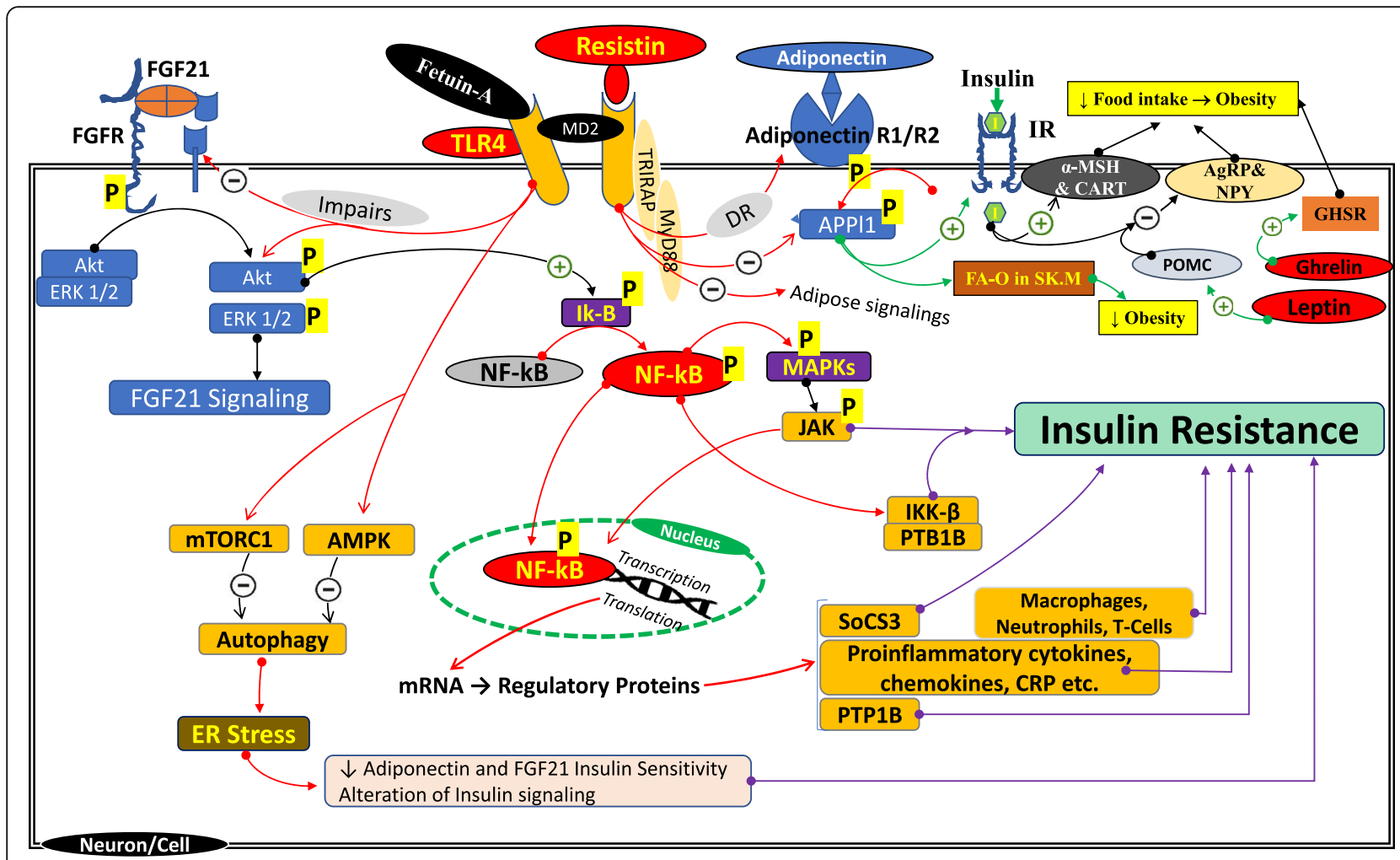

Fig. 4 Role of resistin, TLR4, and NF-kB signalings in developing insulin resistance. $\rightarrow$ : Stimulation/release; $\rightarrow$ : Restore/repair; $\rightarrow$ : Pathway of insulin resistance; $\rightarrow$ : Physiological signalings. $\oplus$ : Stimulation; $\Theta$ : Inhibition. FGF21: Fibroblast growth factor 21; FGFR fibroblast growth factor receptor gene, Akt protein kinase B, ERK extracellular signal-regulated kinases, MD2 myeloid differentiation factor 2, TLR4 Toll-like receptor 4, NFkB nuclear factor kappa-light-chain-enhancer of activated B cells, Ik-B IkB kinase, mTORC1 Mammalian target of rapamycin complex 1, AMPK AMPactivated protein kinase, PI3K phosphatidylinositol-3-kinase, JAK c-Jun N-terminal kinases, MyD88 myeloid differentiation primary response 88, DR diabetic retinopathy, APPI1 adaptor protein, phosphotyrosine interacting, IR insulin resistance, a-MSH \& CART a-melanocyte-stimulating hormone \& cocaine and amphetamine-regulated transcript, AgRP \& NPY Agouti-related protein \& neuropeptide Y, GHSR growth hormone secretagogue receptor, POMC proopiomelanocortin, FA-O in SK.M, skeletal muscle lipid oxidation, SoCS3 suppressor of cytokine signaling 3, PTP1B protein tyrosine phosphatase 1B, ER endoplasmic reticulum

creation and expansion of hypothalamic insulin resistance [63]. The elevated NF- $\kappa B$ signaling in HFD animal models activates the mTORC1, leading to ER (endoplasmic reticulum). This triggers insulin resistance in hypotheses accelerates the obesity and type 2 diabetes progression [64]. Prolonged ER stress leads to increased buildup of stretched protein response which involves insulin resistance and inflammation in the hypothalamus [65].

Fascinatingly, the deficiency of PTP1B in mice improved insulin sensitivity in the central hypothermic region and reduced the progression of obesity and related metabolic complications [66]. TLR family protein (TLR4) also contributes to hypothalamic inflammation (HI) and IR [67]. TLR4 is the main component and target of saturated FAs in the hypothalamus and peripheral tissues that further release the pro-inflammatory mediators, thus triggering the insulin resistance and ER stress in the entire body [67].
Recent findings have reported elevated levels of Fetuin-A, a glycoprotein (TLR4 ligand) released by hepatocytes and adipose cells. Fetuin-Ais needed for FFdependent activation of TLR and facilitates inflammation and IR. TLR4 inhibition in mice protected HFD-induced IR and inflammation [68]. The co-factor protein, myeloid differentiation factor (MyD88) is a downstream mediator for TLR4 pathways in mice, and its reduced levels defended HFD-induced obesity, blood glucose intolerance, and peripheral insulin resistance [69].

Furthermore, some reports have specified that TLR4mediated microglia signaling pathways also have a central part in the control of ARC neural activity and feeding behavior [70]. Finally, this information strongly indicates that the TLR4 path plays a significant role in the progression of overweight, inflammation, and IR. This problem also might be varying with diet variations. The TLR4 is also involved in breast cancer progression [71]. Recent reviews have stated that resistin may 
potentiate the inflammation and IR by activating PLR4 and its downstream pathways, which promote central, peripheral insulin resistance, and inflammation [72].

The resistin released from the adipose tissue in rodents and macrophages in humans can encourage the release of inflammatory mediators and worsen inflammation and IR [73]. Resistin impairs peripheral insulin responsiveness [72], whereas the absence or lack of resistin or administration of antibodies against resistin improved sensitivity to insulin [74], and some reports have described incompatible results [75]. Apart from this contradiction, the resistin has a key role in the invention of pro-inflammatory agents (TNF $\alpha$, LI-6, via NF- $\mathrm{KB}$ ), which are generally involved in peripheral IR in animal models and the humans [76].

Resistin accelerates pro-inflammatory agents (TNF $\alpha$, LI-6, via NF-kB), leading to deep alterations in insulin signaling mechanisms and worsening of IR [77]. Resistin also presents in the hypothalamus and regulates the inflammation in CNS [72], which has modulatory action on neurons and regulates glucose, food consumption, and lipid metabolism. This suggests that resistin has a regulatory role in obesity and IR [77], but the information about resistin-inducing hypothalamic inflammation and its receptors is not well documented.

Resistin may alter insulin receptors, AKT, and ERK1/2 protein phosphorylation in the brain [69]. This might lead to the stimulation of SOCS-3 and PTP1B (negative insulin signaling modulators) [78]. It can also activate JNK and p38 MAPK, which promotes the serine phosphorylation of IRS1. This further increase insulin resistance [60] and another proposed mechanism of resistin-causing hypothalamic inflammation and IR via impairment of adiponectin and FGF21 signaling called as insulin-sensitizing hormones [79]. Resistin treatment decreases adiponectin receptors expression centrally [69]. This protein might be mixed up in adipose-Rs signaling, which contributes to the insulin-sensitizing effect of adiponectin and also diminished FGF21 and its receptors (FGFR1 and KLB) in the hypothalamic region. This further involves the impairment of FGF21 and adiponectin signals in both humans' and animals' (mouse) neural cells [69].

The overexposure of resistin can decrease autophagy via suppression of many autophagy markers like LC3, ATG7, and Beclin1 [80]. This can occur by activating the TLR4 which leads to inhibition of phosphorylation of AMPK and stimulation of Akt/mTOR, which regulates autophagy. In specific, resistin decreased the level of LC3 in the arcuate nucleus through TLR4 signaling [80]. This suggests that resistin/TLR4 or both regulate the neural autophagy and might be a part of inflammation and insulin resistance in the hypothalamic region. This observation is remarkable about HFD-induced stimulation of astrocytes and microglia that are crucially involved in inflammation and IR in the hypothalamic region [81].

\section{Conclusion}

Obesity plays a vital key role in the development of central and fringe insulin resistance leading to type 2 diabetes. The resistin, TLR4, and NF- $\mathrm{kB}$ signaling mechanisms promote the central inflammation and insulin resistance.

The HFD-STZ diabetic model has become more popular for the assessment of new phytochemicals against type 2 diabetic complications. In this combination, the HFD develops obesity and insulin resistance (IR) and the streptozotocin can cause an insulin shortage by DNA fragmentation and induce oxidative stress in the $\beta$ cells by accelerating the invention of ROS like hydroxyl, nitrogen oxide, hydrogen peroxide, and oxygen.

$\mathrm{NF}-\mathrm{kB}$ is a crucial pathway in the pathogenesis of various macro- and micro-level diabetic complications. Inhibition of the NF- $\mathrm{kB}$ signaling cascade may provide an effective clinical correlation for diabetes to prevent complications. Although various natural, synthetic, and semi-synthetic NF- $\mathrm{KB}$ inhibitors are available, their clinical considerations are limited. Based on this review, it can be concluded that the resistin, TLR4, and NF- $\mathrm{KB}$ signaling pathways are highly focused and are the vital future targets for overcoming the central, fringe changes, and effective treatment of diabetic vascular complications.

\section{Abbreviations}

ADP: Adenosine diphosphate; AGEs: Advanced glycation end products; AgRP \& NPY: Agouti-related protein \& neuropeptide Y; Akt: Protein kinase B; AMPK: AMP-activated protein kinase; APPI1: Adaptor protein, phosphotyrosine interacting; ARase: Aldose reductase; AT-II: Angiotensin II; ATP: Adenosine triphosphate; ATP: Adenosine tri-phosphate; BCl-2: B cell lymphoma 2; BG: Blood glucose; BGL: Blood glucose level; BP: Blood pressure; CAM: Cellular adhesion molecule; COX-2: Cyclooxygenase-2; CYP: Cytochrome P450; DAG: Diacylglycerol; DN: Diabetic nephropathy; DNA: Deoxyribonucleic acid; eNOS: Endothelial NOS; ER: Endoplasmic reticulum; ERK: Extracellular signal-regulated kinases; ET-1: Endothelin 1; FGF21: Fibroblast growth factor 21; FGFR: Fibroblast growth factor receptor gene; GHSR: Growth hormone secretagogue receptor; GLUT2: Glucose transporter 2; GSH: Glutathione; HbAc1: Glycated hemoglobin; HFD: High-fat diet; ICAM: Intercellular adhesion molecule; IK-B: IKB kinase; IL-1: Interleukin-1; IL-6: Interleukin-6; IL-8: Interleukin-8; iNOS: Inducible nitric oxide synthase; IR: Insulin resistance; IR: Insulin resistance; JNK: c-Jun N-terminal kinase; KLB: $\beta$-Klotho gene; LC3: Light chain 3; MAPK: Mitogen-activated protein kinase; MCP-1: Monocyte chemoattractant protein-1; MD2: Myeloid differentiation factor 2; mTORC1: Mammalian target of rapamycin complex 1; MYD88: Myeloid differentiation primary response 88; NAD: Nicotinamide adenine dinucleotide; NADPH: Nicotinamide adenine dinucleotide phosphate; NCV: Nerve conduction velocity; NF-kB: Nuclear factor kappalight-chain-enhancer of activated B cells; NF-KB: Nuclear factor kappa-lightchain-enhancer of activated B cells; NGF: Nerve growth factor; NO: Nitric oxide; PI3K: Phosphatidylinositol-3-kinase; PKC: Protein kinase C;

POMC: Proopiomelanocortin; PTP1B: Protein tyrosine phosphatase 1B; RAGE: Receptor for advanced glycation endproducts; ROS: Reactive oxygen species; SoCS3: Suppressor of cytokine signaling 3; STZ: Streptozotocin; TG: Triglycerides; TLR: Toll-like receptor 4; TNF: Tumor necrosis factor beta; TNF-a: Tumor necrosis factor-alpha; VEGF: Vascular endothelial growth factor; XO: Xanthine oxidase; ZDF: Zucker diabetic fatty; a-MSH \& CART: aMelanocyte-stimulating hormone \& cocaine and amphetamine-regulated transcript 


\section{Acknowledgements}

We are very much grateful to Miss Shalini Gudise for the technical support.

\section{Authors' contributions}

VG performed all the research activities, writing, and design. BC conceived the study and participated in its design and coordination. The authors have read and approved the manuscript.

\section{Funding}

This research did not receive any specific grant from funding agencies in the public, commercial, or not-for-profit sectors.

\section{Availability of data and materials}

Not applicable

\section{Ethics approval and consent to participate}

Not applicable

\section{Consent for publication}

Not applicable

\section{Competing interests}

The authors declare that they have no competing interests.

\section{Author details}

'Department of Pharmacology, SSJ College of Pharmacy, Vattinagulapally, Gandipet, Hyderabad, Telangana 500075, India. ${ }^{2}$ Department of Pharmacology, Roland Institute of Pharmaceutical Sciences, Berhampur, Odisha 760010, India.

\section{Received: 9 July 2020 Accepted: 30 September 2020}

Published online: 17 December 2020

\section{References}

1. Annette B, Ramesh K, Hemachandra Reddy P (2017) Dynamics of diabetes and obesity: epidemiological perspective. Biochimica et Biophysica Acta. 1863:1026-1036

2. WHO (2020) Diabetes. Word health organization. https://www.who.int/newsroom/fact-sheets/detail/diabetes

3. IDF (2020) Diabetes facts \& figures. International Diabetes Federation. 9th edition. https://www.idf.org/aboutdiabetes/what-is-diabetes/facts-figures. html

4. Chang WC, Wu JSB, Chen CW, Kuo PL, Chien HM, Wang YT, Shen SC (2015) Protective effect of vanillic acid against hyperinsulinemia, hyperglycemia and hyperlipidemia via alleviating hepatic insulin resistance and inflammation in high-fat diet (HFD)-fed rats. Nutrients. 7(12):9946-9959

5. Kristina B, Fran Stjepan N, Ivan K, Ivana PR (2018) Treatment approach to type 2 diabetes: Past, present and future. World J Diabetes. 15: 9(12):209219

6. Yang-wei W, Guang-dong S, Jing S, Shu-jun L, Ji W, Xiao-hong X, Li-ning M (2013) Spontaneous type 2 diabetic rodent models. J Diabetes Res. 2013 401723

7. Gaurav G, Ridhima W, Parijat P, Sachin Kumar S, Monica G, Saurabh S (2020) Obesity and diabetes: pathophysiology of obesity-induced hyperglycemia and insulin resistance. Pathophysiology of Obesity-Induced Health Complications. pp 81-97

8. Nasimeh V, Farzaneh R, Ahmad RS, Sharifeh K, Gholamreza D, Gilda E (2018) Novel high-fat diet formulation and streptozotocin treatment for induction of prediabetes and type 2 diabetes in rats. Adv Biomed Res. 7:107

9. Chinedum OE, Kate CE, Sonia C, Udeme NE (2013) Review of the mechanism of cell death resulting from streptozotocin challenge in experimental animals, its practical use and potential risk to humans. J Diabetes Metab Disord. 12:60

10. Abdulrahman MS, Hannah MB, Arun VED, Tiffany CYT, Lindsay MP, Jeremy GT (2018) The effect of streptozotocin-induced hyperglycemia on N-and Olinked protein glycosylation in mouse ovary. Glycobiology. 28(11):832-840

11. Philipp AG, Guy AR (2017) The role of oxidative stress and hypoxia in pancreatic beta-cell dysfunction in diabetes mellitus. Antioxid Redox Signal. 26(10):501-518
12. Gvazava IG, Rogovaya OS, Borisov MA, Vorotelyak EA, Vasiliev AV (2018) Pathogenesis of type 1 diabetes mellitus and rodent experimental models. Acta Naturae. 10(1):24-33

13. Wu M-Y, Giou-TY T-TL, Chia JL (2018) The oxidative stress and mitochondrial dysfunction during the pathogenesis of diabetic retinopathy. Oxid Med Cell Longev. 2018:1-13

14. Arwa MT, Nahdi A, Annie J, Haider R (2017) Elucidation of molecular mechanisms of streptozotocin-induced oxidative stress, apoptosis, and mitochondrial dysfunction in Rin-5F pancreatic $\beta$-cells. Oxid Med Cell Longev. 2017:7054272

15. Kahraman S, Aydin C, Elpek GO, Dirice E, Sanlioglu AD (2015) Diabetesresistant NOR mice are more severely affected by streptozotocin compared to the diabetes-prone NOD mice: correlations with liver and kidney GLUT2 expressions. J Diabetes Res. 2015:450128

16. Nidal AQ, Adnan AB (2015) Impact of streptozotocin on altering norma glucose homeostasis during insulin testing in diabetic rats compared to normoglycemic rats. Drug Des DevelTher. 9:2515-2525

17. Jiali L, Lina H, Leilei Z, Yerong Y (2016) Free fatty acids, not triglycerides, are associated with non-alcoholic liver injury progression in high fat diet induced obese rats. Lipids Health Dis. 15:27

18. Snel M, Jonker JT, Schoones J, Lamb H, de Roos A, Pijl H (2012) Ectopic fat and insulin resistance: pathophysiology and effect of diet and lifestyle interventions. Int J Endocrinol. 2012:983814

19. Sotirios T, Alexios SA, Evangelos O, George-Aggelos P, Georgia V, Spyridon P (2019) The role of inflammation in diabetes: current concepts and future perspectives. Eur Cardiol. 14(1):50-59

20. Konstantinos P, Maciej B, Eleni B, Manfredi R, Michael E (2018) Complications of diabetes 2017. Diabetes Res. 2018:3086167

21. Valeska O, Soumyalekshmi N, Omar E, Claudio A, Carlos S, Felipe A (2018) Association between insulin resistance and the development of cardiovascular disease. Cardiovasc Diabetol. 17:122

22. Tobon VJC, Cuevas E, Torres RMA (2014) CNS Neurol. Disord Drug Targets 13(9):1615-1626

23. Xing L, Gao HL, Dai GY, Sun HM, Xu HQ (2016) Food advanced glycation end products aggravate the diabetic vascular complications via modulating the AGEs/RAGE pathway. Chin J Nat Med. 14(11):844-855

24. Mitra T, Dilek GY, Abd AT, Dinesh S, Frank LB, Hassan F (2017) Diabetic neuropathy: current status and future prospects. journal of diabetes research. 2017:1-2

25. Aslam A, Singh J, Rajbhandari S (2014) Pathogenesis of painful diabetic neuropathy. Pain Res Treat. 2014:412041

26. Chun-Pai Y, Cheng-Chieh L, Chia-Ing L, Chiu-Shongn L (2015) Cardiovascular risk factors increase the risks of diabetic peripheral neuropathy in patients with type 2 diabetes mellitus. Medicine (Baltimore). 94(42):e1783

27. Sachin V, Suryavanshi, YA, Kulkarni (2017) NF-kß: a potential target in the management of vascular complications of diabetes. Front Pharmacol. 8:798.

28. Su Bin C, Won SE, Min JS, Hyun JK, Jung HP, Yeon JC (2019) Transduced tataldose reductase protects hippocampal neuronal cells against oxidative stress-induced damage. Exp Neurobiol. 28(5):612-627

29. Chunkui Z, Limin W, Fengming N, Wei J, Jiang W, Hongliang Z (2014) Critical illness polyneuropathy and myopathy: a systematic review. Neural Regen Res. 9(1):101-110.

30. Lara K, Melanie JM, Anna JV, Anna LO (2019) Obesity-induced TNFa and IL-6 signaling: the missing link between obesity and inflammation-driven liver and colorectal cancers. Cancers (Basel). 11(1):24

31. Shreena JD, Ben P, Avraham R (2018) Mechanisms of phytonutrient modulation of Cyclooxygenase-2 (COX-2) and inflammation related to cancer. Nutr Cancer. 70(3):350-375

32. Wei C, Tuo Y, Huan L, Lijuan H, Kai Z, Xiaoming H (2019) Peroxisome proliferator-activated receptor $\gamma$ (PPARY): a master gatekeeper in CNS injury and repair. Prog Neurobiol. 163(164):27-58

33. Frederik P (2011) Peter R (2018) Diagnosis of diabetic kidney disease: state of the art and future perspective. Kidney Int Suppl 8(1):2-7

34. Pérez-Morales RE, del Pino MD, Valdivielso JM, Ortiz A, Mora-Fernández C, NavarroGonzález JF (2019) Inflammation in diabetic kidney disease. Nephron. 143:12-16

35. Javier DC, Desirée Luis R, Ernesto MN, Víctor GT, Carolina HC, Carla F (2020) Inflammatory targets in diabetic nephropathy. J Clin Med. 9(2):458

36. Haisong Z, Shao CS (2015) NF-kB in inflammation and renal diseases. Cell Biosci. 5:63

37. Mariana RS, van der EM H, Lie Mulder $H$, Jolanda MS, Izabela A, Rodenhuis Z (2016) Mechanism and role of MCP-1 upregulation upon chikungunya virus infection in human peripheral blood mononuclear cells. Sci Rep. 6:32288 
38. Patrick L, Pascal C, Veronique R, Edward GL, Mingyi W (2015) Chapter 2 cellular and molecular determinants of arterial aging. Early Vascular Aging (EVA). 2015:7-16

39. Ning S, Friedrich T, Linlin G (2019) NFKB and kidney injury. Front Immunol. 10:815

40. Nyasha C, Ophius M, Hilda M (2013) Microalbuminuria in patients with chronic kidney disease at Parirenyatwa Hospital in Zimbabwe. Pan Afr Med J. $14: 39$

41. Meng XM, Tang PM, Li J, Lan HY (2015) TGF- $\beta /$ Smad signaling in renal fibrosis. Front Physiol. 6:82

42. Temitope IA, LeiDu MH, Xueyan Z, QianX U, Chowdhury A, Ying S (2020) Keap1/Nrf2/ARE signaling unfolds therapeutic targets for redox imbalancedmediated diseases and diabetic nephropathy. Biomed Pharmacother. 123: 109732

43. Martin MN, Michael WU (2015) Diabetic retinopathy - ocular complications of diabetes mellitus. World J Diabetes. 6(3):489-499

44. Zhang K, Ferreyra HA, Grob S, Bedell M, Zhang JJ (2012) Diabetic retinopathy: genetics and etiologic mechanisms. In: D.R. Hinton, 5th edn. Elsevier, London

45. Musat O, Cernat C, Labib M, Gheorghe A, Toma O, Zamfir M (2015) Diabetic macular edema. Rom J Ophthalmol. 59(3):133-136

46. Ryan L, Tien YW, Charumathi S (2015) Epidemiology of diabetic retinopathy, diabetic macular edema and related vision loss. Eye Vis (Lond) 2:17

47. Safi SZ, Qvist R, Kumar S, Batumalaie K, Ismail IS (2014) Molecular mechanisms of diabetic retinopathy, general preventive strategies, and novel therapeutic targets. Biomed Res Int. 2014:801269

48. Małgorzata N, Andrzej G (2016) The role of the reactive oxygen species and oxidative stress in the pathomechanism of the age-related ocular diseases and other pathologies of the anterior and posterior eye segments in adults. Oxid Med Cell Longev. 2016:3164734

49. Shinwan K, Jan TV, Borna R (2019) Cytokines in inflammatory disease. Int J Mol Sci. 20(23):6008

50. Noboru T, Shinichi T, Sadanobu N (2011) Nitric oxide-mediated coronary flow regulation in patients with coronary artery disease: recent advances. Int J Angiol. 20(3):121-134

51. Sayon R, Shruti A, Sumon R (2016) Retinal fibrosis in diabetic retinopathy. Exp Eye Res. 142:71-75

52. Kowluru RA, Zhong Q, Santos JM (2012) Matrix metalloproteinases in diabetic retinopathy: potential role of MMP-9. Expert. Opin Invest Drugs. 21(6):797-805

53. Kowluru RA, Shan Y, Mishra M (2016) Dynamic DNA methylation of matrix metalloproteinase-9 in the development of diabetic retinopathy. Lab Invest. 96(10):1040-1049

54. Vinay K, Abul A, Jon A (2014) Robbins \& cotran pathologic basis of disease pathological basis of disease. $10^{\text {th }}$ edition

55. Gregor MF, Hotamisligi GS (2011) Inflammatory mechanisms in obesity. Annu Rev Immunol. 29:415-445

56. Yacir B, Mohammed T (2019) Molecular mechanisms underlying obesityinduced hypothalamic inflammation and insulin resistance: pivotal role of resistin/TLR4 pathways. Front Endocrinol (Lausanne) 10:140

57. Linlin C, Huidan D, Hengmin C, Jing F, Zhicai Z, Junliang D (2018) Inflammatory responses and inflammation-associated diseases in organs. Oncotarget. 9(6):7204-7218

58. Jais A, Brüning JC (2017) Hypothalamic inflammation in obesity and metabolic disease. J Clin Invest. 127(1):24-32

59. Estefania BM, Zaida AJ, Aranzazu MM, Francesca I, Irene EL, Sandra LD (2019) Relationship between oxidative stress, ER stress, and inflammation in type 2 diabetes: the battle continues. J Clin Med. 8(9):1385

60. Copps KD, White MF (2012) Regulation of insulin sensitivity by serine/ threonine phosphorylation of insulin receptor substrate proteins IRS1 and IRS2. Diabetologia. 55(10):2565-2582

61. Chen Y, Hung-Yun L, Ning-Chun L, Janet DS, Shuyuan Y, Lei-Ya F (2013) Neuronal androgen receptor regulates insulin sensitivity via suppression of hypothalamic NF-kB-mediated PTP1B expression. Diabetes. 62(2):411-423

62. Santiago V, Julie CK, Tamera B, Dae YJ, Jason KK, Roger JD (2013) Dietinduced obesity mediated by the JNK/DIO2 signal transduction pathway. Genes Dev. 27(21):2345-2355

63. Douglass JD, Dorfman MD, Fasnacht R, Shaffer LD, Thaler JP (2017) Astrocyte IKKB/NF-KB signaling is required for diet-induced obesity and hypothalamic inflammation. Mol Metab 6(4):366-373
64. Zhuo F, Elizabeth RG, Dongmin L (2013) Regulation of insulin synthesis and secretion and pancreatic beta-cell dysfunction in diabetes. Curr Diabetes Rev. 9(1):25-53

65. Fernanda G, Felice D, Sergio T (2017) Getting a "GRiP" on hypothalamic endoplasmic reticulum stress to combat obesity. Diabetes. 66(1):17-19

66. Aberdein N, Dambrino RJ, Carmo JM, Wang Z, Mitchell LE, Drummond HA (2018) Role of PTP1B in POMC neurons during chronic high-fat diet: sex differences in regulation of liver lipids and glucose tolerance. Am J Physiol Regul Integr Comp Physiol. 314(3):R478-R488

67. Rogero MM, Calder PC (2018) Obesity, inflammation, Toll-like receptor 4 and fatty acids. Nutrients. 10(4):E432

68. Pal D, Dasgupta S, Kundu R, Maitra S, Das G, Mukhopadhyay S (2012) Fetuin-A acts as an endogenous ligand of TLR4 to promote lipid-induced insulin resistance. Nat Med 18(8):1279-1285

69. Benomar Y, Amine H, Crépin D, Rifai S, Riffault L, Gertler A (2016) Central resistin/TLR4 impairs adiponectin signaling, contributing to insulin and FGF21 resistance. Diabetes. 65(4):913-926

70. Reis WYC, Gao Y, Tschöp MH (2015) Brain innate immunity regulates hypothalamic actuate neuronal activity and feeding behavior. Endocrinology 156(4):1303-1315

71. Wang CH, Wang PJ, Hsieh YC, Lo S, Lee YC, Chen YC (2018) Resistin facilitates breast cancer progression via TLR4-mediated induction of mesenchymal phenotypes and stemness properties. Oncogene. 37(5):589600

72. María R, Cristina P, Eduardo M, Nilda G, Carmen M, Fernández M (2018) Central s-resistin deficiency ameliorates hypothalamic inflammation and increases whole body insulin sensitivity. Sci Rep. 8:3921

73. Maggie SB, Derek KH, Jessica NK, Kelsey AS, Mario K (2018) Contribution of adipose tissue inflammation to the development of type 2 diabetes mellitus. Compr Physiol. 9(1):1-58

74. Md SJ, Sarah MW, Qizhi Y, Changyi C (2012) Resistin: functional roles and therapeutic considerations for cardiovascular disease. Br J Pharmacol. 165(3): 622-632

75. Mostafazadeh M, Haiaty S, Rastqar A, Keshvari M (2018) Correlation between resistin level and metabolic syndrome component: a review. HormMetab Res. 50(7):521-536

76. Pine GM, Batugedara HM, Nair MG (2018) Here, there and everywhere: resistin-like molecules in infection, inflammation, and metabolic disorders. Cytokine. 110:442-451

77. Hannan AF, Culligan KG (2015) Human resistin and the RELM of inflammation in diabesity. Diabetol Metab Syndr. 7:54

78. Jérémie B, André KC, Ronald K (2014) Insulin receptor signaling in normal and insulin-resistant states. Cold Spring Harb Perspect Biol. 6(1):a009191

79. Cheng KK, Lam KS, Wang B, Xu A (2014) Signaling mechanisms underlying the insulin-sensitizing effects of adiponectin. Best Pract. Res Clin Endocrinol Metab. 28(1):3-13

80. Miao J, Benomar Y, Rifai S, Poizat G, Riffault L, Crépin D, Taouis M (2018) Resistin inhibits neuronal autophagy through Toll-like receptor 4. J Endocrinol. 238(1):77-89

81. André C, Guzman QO, Rey C, Rémus-Borel J, Clark S, Castellanos JA (2017) Inhibiting microglia expansion prevents diet-induced hypothalamic and peripheral inflammation. Diabetes. 66(4):908-919

\section{Publisher's Note}

Springer Nature remains neutral with regard to jurisdictional claims in published maps and institutional affiliations. 